U.S.A. and in Japan, would yield slightly lower mortality rates even for the same incidence of disease, ${ }^{10}$ but would not affect the relative size of the incidence rates observed in the national cancer survey. A dietary hypothesis (rather than a genetic hypothesis) might be invoked to explain the differential synthesis of the anticarcinogen oestriol versus the carcinogens oestrone and oestradiol. ${ }^{911}$ As the present generation born in the U.S.A. ages and as future generations adopt more Western patterns of fertility and diet, important leads may be gained into the aetiological importance of heredity and environmental factors in breast cancer.

\footnotetext{
${ }^{1}$ Haenszel, W. H., and Kurihara, M., Fournal of the National Cancer Institute, 1968, 40, 43 .

Petrakis, N. L., Science, 1971, 173, 347.

3 Smith, R. L., fournal of the National Cancer Institute, 1957, 18, 385

4 Bulbrook, R. D., Thomas, B. S., and Utsunomiya, J., Nature, 1964, 201, 189.

MacMahon, B, et al, Lancet, 1971, $2,900$.

6 Buell, P., Fournal of the National Cancer Institute, 1973, 51, 1479

${ }^{6}$ Buell, P., Fournal of the National Cancer Institute, 1973, 51, 1479.

7 Kamoi, M., Tohoku fournal of Experimental Medicine,

MacMahon, B., Cole, P., and Brown, J., Fournal of the National Cancer Institute, 1973, 50, 21 .

10 Wynder, E. L., et al., Surgery, Gynecology and Obstetrics, 1963, 117, 196.

11 Lea, A. J., Lancet, 1966, 2, 332.
}

\section{Modifications to the Breasts}

The normal size of women's breasts is a matter of current fashion and personal taste, and deviation from this norm is tolerated or not depending on the degree of deviation and the emphasis placed upon it by the person in question.

Breasts which are too big usually result from benign pubertal, puerperal, or menopausal hypertrophy. The patients complain of embarrassment, difficulty in obtaining well-fitting underwear, intertrigo, thoracic back pain, difficulty in sleeping, and occasionally of a dragging pain in the breasts. The shoulders may be grooved and pigmented by the supporting garments. Such large breasts require reduction mammaplasty, in which the surgeon resects the breast tissue and repositions the nipple. The method selected will depend on the likelihood that the woman will want to breast-feed in a subsequent pregnancy, the size of the breast, and the technical preferences of the surgeon. One inevitable sequel is scarring, usually situated circumferentially around the nipple, downwards to and transversely in or above the submammary line. Postoperatively there is usually permanent reduction or absence of nipple sensation. Since the nipple is in some methods transposed on a vascular pedicle there is also a risk of partial or total loss of the nipple. Most patients when warned of these hazards and disadvantages of surgery will accept them in order to achieve relief from their symptoms.

Absence of one or both breasts may be congenital or result from mastectomy. Construction or reconstruction of the absent breast may be possible, depending on the laxity of the skin. The methods used for reconstruction are the same as for augmentation. The nipple, if absent, may be fashioned using a partial thickness graft from the contralateral side or wholethickness skin from the labia minora (which have skin of the most closely matching texture and colour). Immediate reconstruction of the breast after subcutaneous mastectomy for breast diseases is now performed for carefully selected cases.

Patients with small breasts complain of social withdrawal or depression, which they ascribe to their defect. It is important to establish the exact cause of their symptoms before embarking on surgery. Examination often shows a stooping, round- shouldered stance adopted by the patient to disguise the defect.

The breast can be augmented in a plane between the pectoral fascia and the breast tissue, placing the normal breast tissue between the augmenting material and the skin, when a more normal "feeling" is achieved. Either autogenous lipodermal grafts or prostheses are used to achieve the augmentation. The lipodermal grafts are taken from the buttocks and the bilateral $20 \mathrm{~cm}$ scars are sited within the bikini line. Some $10 \%$ or more of such transplants fail totally, while in the remainder about $10 \%$ of the transplanted tissue is absorbed. Since the tissue available for transplantation and that which may be expected to survive as a free graft is limited, so too is the degree of possible augmentation. Fibrosis or calcification of the transplant sometimes occurs, giving a hard breast. In those cases which are satisfactory, however, patients and their husbands are sometimes happier knowing that the augmented breast consists of their own tissue.

Prosthetic augmentation has been proved safe. There is no higher incidence of malignant disease of the breast in cases so treated than in the untreated. So far sponges of various materials have proved unsatisfactory. The best prostheses are filled and sealed or inflatable envelopes of silicone rubber, containing either soft silicone rubber, dextran, or other solution. These feel almost normal, give a good contour, and are easy to insert. The operation takes less time than lipodermal augmentation and requires fewer days of inpatient care. The prostheses are not cheap. Some have to be removed because of haematoma, infection, or wound breakdown, despite all precautions to prevent these complications. A few cases develop a hard capsule of fibrous tissue around the prosthesis.

Asymmetrical breasts may be corrected by augmentation of the smaller or reduction of the larger by the methods described above; but transfer of tissue from one breast to the other meets with limited success. Pendulous breasts may follow pregnancy or be a result of ageing. If sufficient breast tissue remains, a reduction of mammary skin may be performed, using remaining skin as a sort of brassiére to contain and support the existing breast. The nipple is repositioned at a normal level. If, because of hypoplasia, the breast is ptotic without redundant skin, then augmentation may be expected to restore a normal contour.

The N.H.S. makes provision for the treatment of patients suffering from psychological or physical disease and surgery to the breast may come under either head. However, surgery of this sort may expect to receive a lower priority for admission than that for cancer, trauma, and other severe diseases.

\section{After the Anger}

"Ban Private Beds"-“B.M.A. Hypocrites," declared some amateur looking posters outside the City Hall, Kingston upon Hull, last week. The handful of political student protestors picketing the Representatives at the start of the Friday morning session were unlikely to have made any converts. Nevertheless, the demonstration served to remind doctors that the private beds dispute ${ }^{1}$ could well be used by politicians to divert attention from the real dilemma-"what to do about the N.H.S."

By her inept handling of the dispute Mrs. Barbara Castle united the profession and concentrated doctors' minds to an extent that is unparalleled since 1948. The depth of the discontent among staff and their disillusion with the way in which 